\title{
First report of actinidia virus $A$ and $B$ infecting kiwifruit in Jiangxi province in China
}

\author{
Man Fei Zou ${ }^{1} \cdot$ Ming Feng Yan ${ }^{1} \cdot$ Ying Zhou ${ }^{1} \cdot$ Yuan Xiu Wang ${ }^{1} \cdot$ Gui Hong Xiong ${ }^{1} \cdot$ Jun Xi Jiang ${ }^{1}$
}

Received: 24 January 2019 / Accepted: 3 October 2019 / Published online: 10 November 2019

(C) Società Italiana di Patologia Vegetale (S.I.Pa.V.) 2019

Keywords Kiwifruit · Actinidia virus A $\cdot$ Actinidia virus $B \cdot$ RT-PCR

In recent years, Actinidia virus $A$ and $B$ (AcVA and AcVB), both of which belong to the genus Vitivirus of the family Betaflexiviridae, have been reported in New Zealand, South Korea and Hubei, China (Blouin et al. 2012; Cho et al. 2017; Zheng et al. 2014). During investigation on field disease of kiwifruit in June 2018 in Jiangxi Province of eastern China, symptoms of leaf chlorosis, ringspots and deformities were seen, suggesting the presence of a virus. Therefore, testing was carried out for a range of viruses including AcVA and AcVB. Total RNA were extracted from the infected kiwifruit leaves and subjected to RT-PCR for detecting AcVA using primer sets AcVA5F/5R and AcVA CP NTR F/R, and AcVB using primer sets AcVB5F/Viti3'R and AcVB CP NTR F/R (Blouin et al. 2012; Cho et al. 2017), respectively. Primer sets AcVA5F/5R and AcVB5F/Viti3'R target the RNA-binding protein genes of AcVA and AcVB, while primers AcVA CP NTR F/R and AcVB CP NTR F/R amplify their regions covering partial movement protein, full coat protein and partial RNA binding protein genes. Results showed that $100 \%$ and $68.6 \%$ of the tested samples were infected with AcVA and AcVB, respectively. From AcVA, a 283 bp long sequence of the RNA-binding protein gene (GenBank accession No. MH732945) and a 597 bp long sequence of the complete CP gene (MK729781) were determined, which shared $92.9 \%$ and $90.8 \%$ homology with the corresponding

Jun Xi Jiang

jxau2011@126.com

1 College of Agronomy, Jiangxi Agricultural University, Nanchang 330045, China sequence of AcVA isolate in New Zealand (JN427014). From $\mathrm{AcVB}$, a $369 \mathrm{bp}$ long sequence of the RNA-binding protein gene (MH732946) and a 597 bp long sequence of the complete CP gene (MK729782) were also determined, and they shared $99.2 \%$ and $98.7 \%$ homology with the corresponding sequence of New Zealand AcVB isolate (JN427015). The $369 \mathrm{bp}$ sequence was also compared with the corresponding sequence of AcVB isolate (accession No. KJ696778) in Hubei Province, which is adjacent to Jiangxi Province, and they shared only $88.1 \%$ homology, and the homology between Jiangxi and Hubei isolates is less than that between Jiangxi and New Zealand isolates. To our knowledge, this is the first report of AcVA and AcVB infecting kiwifruit in Jiangxi province in China.

Funding information This study was supported by the National Natural Science Foundation of China (Grant No. 31460452).

\section{References}

Blouin AG, Chavan RR, Pearson MN, MacDiarmid RM, Cohen D (2012) Detection and characterisation of two novel vitiviruses infecting Actinidia. Arch Virol 157(4):713-722

Cho SY, Kim H, Yi SI (2017) First report of Actinidia virus A and B infecting Actinidia chinensis in South Korea. Plant Dis 101(8): $1560-1560$

Zheng YZ, Wang GP, Hong N et al (2014) First report of Actinidia virus A and Actinidia virus B on kiwifruit in China. Plant Dis 98(11): $1590-1590$

Publisher's note Springer Nature remains neutral with regard to jurisdictional claims in published maps and institutional affiliations. 